\title{
Location-grouping algorithm based on limited actuators deformable mirror for high precision wavefront aberration correction in adaptive optics system
}

\author{
Meng Yan ${ }^{1}$, Lei Huang ${ }^{1 * 2 *}$, Qi Bian, Chenlu Zhou ${ }^{1}$, Xingkun Ma ${ }^{1}$, Mali Gong ${ }^{1}$ \\ 1 Department of Precision Instruments, Tsinghua University, Beijing 100084, China \\ 2 College of Optical Sciences, University of Arizona, 1630 East University Boulevard, Tucson, AZ \\ 85721, USA \\ hl@tsinghua.edu.cn, leihuang@email.arizona.edu
}

\begin{abstract}
The aberration in the center position of wavefront can be corrected well when the deformable mirrors (DM) used in highresolution adaptive optics system of telescope. However, for the defocus and spherical aberration of telescope, the four corners of the wavefront cannot be corrected well. A novel correction method with different levels and regions of deformable mirror is proposed to solve this problem. The control elements of wavefront in four corners are divided. And every four or five DM units in one corner is in a group. Compared with conventional correction method, the locationgrouping method showed significant advantages in correction of different order aberrations.
\end{abstract}

\section{INTRODUCTION}

Adaptive optics was originally proposed to improve the dynamic effects of the atmospheric turbulence in astronomical telescope observations. After decades of development, it has been widely used in astronomical field, retinal imaging and laser-based nuclear fusion [1]. Many modern astronomical telescopes use adaptive system to correct the wavefront aberration caused by various factors in real time. The deformable mirror (DM) is the main device in adaptive optics system and it corrects the aberration by adjusting the mirror surface.

With the development of the DM and upgrading the algorithm, the correction effect of the adaptive optics system on the wavefront aberration is gradually improved and most of Zernike mode aberration [2] can be corrected well. However, the four corners of the corrected wavefront cannot get a good consistency. Especially the defocus and spherical aberration have great influence on the correction of the four corners of wavefront. It is a good way to abandon the edge area of wavefront and only use the central part. And increasing the number of actuator units of deformable mirror or changing the structure of adaptive optic system are also good ways to solve this problem. However, it will result in low utilization of light energy and cost increasing in traditional ways. Optimizing algorithm is another way to solve this problem. The aberration correction procedure can be divided into two steps, and a novel correction method at different levels and regions of deformable mirror is proposed to solve this problem.

\section{SUB-REGIONAL WAVEFRONT CORRECTION METHOD}

\subsection{Principle of reflective deformable mirror}

The deformable mirror (DM) is the main device in adaptive optics system. The principle of reflective deformable mirror is that the random disturbance voltage makes the DM mirror surface change by the formula (1).

$$
S=\sum_{i=1}^{N} u_{i} l_{i}(x, y)
$$


Where $u_{i}$ is the disturbance voltage of the $i$ th DM unit, and $l_{i}$ is the response function of the $i$ th DM. $N$ is the unit number and $S$ is the surface of DM mirror.

Assume that the target surface is $E$, we can calculate voltage by the formula (2).

$$
F U=E
$$

Where the $F$ is the response function, which can be obtained by the actual measurement of each actuator unit. $\mathbf{E}$ is the target surface, which can be measured by Hartmann-Shack wavefront sensor. Then the voltage of DM can be calculated. But in general it is difficult to find the $U_{0}$ precisely, there is the residual error of the wavefront. So getting the proper $U_{0}$ is the main purpose of the optimization algorithm, and this paper is based on the above.

\subsection{Spherical aberration and defocus characteristics}

In optometry and ophthalmology, the Zernike polynomials are used to describe aberration of cornea or lens from an ideal spherical shape, which results in refraction errors. An arbitrary wavefront is a liner combination of infinite fundamental wavefront. The Zernike polynomials can be used to decompose arbitrary aberrations, and represent a specific type of aberration such as defocus, spherical aberration, coma and astigmatism.

Three typical aberrations (defocus, $45^{\circ}$ astigmatism, spherical aberrations) [3] were taken as the target aberrations to be corrected by LS (Least Square) algorithm and the results are listed in Fig. 1. The first column is the original aberrations and the second column is the residual error after corrections by LS algorithm. It can be seen from the results that after optimized residual error of the central surface is relative less and the root-mean-square (RMS) ratio of residual error of the central surface decreased obviously. However, the result shows that the residual error of the four corners is not obvious after the correction and the RMS or PV is large.
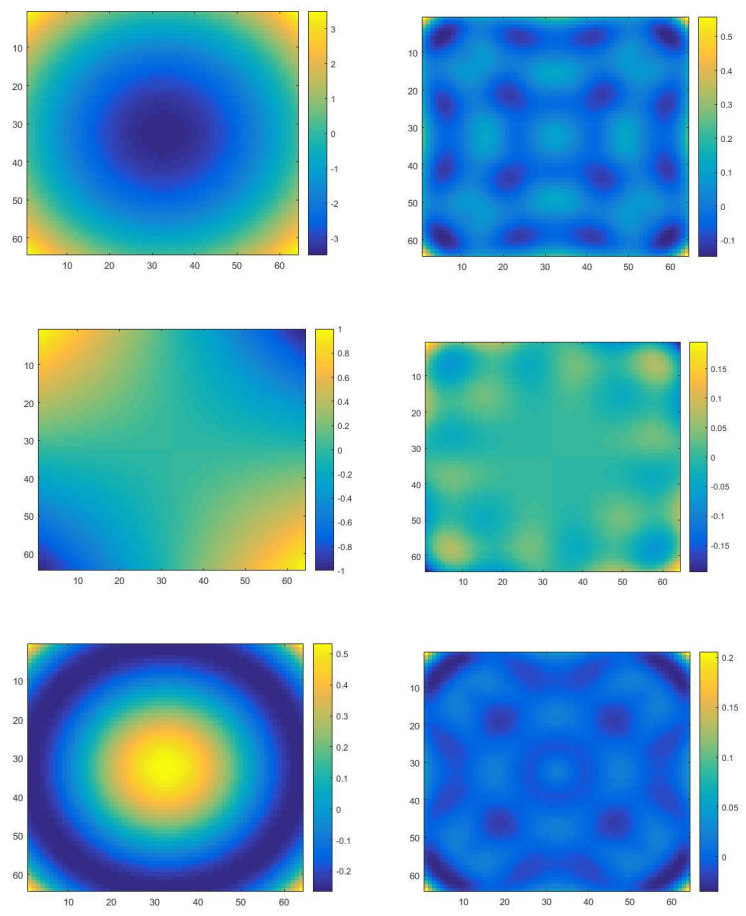

Figure 1. Defocus, $45^{\circ}$ astigmatism, spherical aberrations and correct

Most of the correction algorithms are currently used and the deformable mirror can correct wavefront aberration in a certain extent. There are enough DM units in the middle of mirror and the distribution is also comparatively homogeneous, so they are able to work together to achieve a good optimization effect. The DM units of the edge part are less, and there are less units in the four vertexs of the mirror. So the aberration in the four corners of wavefront is not 
able to be corrected well. Increasing the number and changing the position of the DM units will improve weaknesses. In this way it will increase the cost and schedule. Another way to solve this problem is to optimize the algorithm without changing the structure of DM.

\subsection{Principle of Sub-regional wavefront correction method}

According to the characteristics of various aberrations (defocus, astigmatism, sphere), it is a good way to correct the corner aberration on the basis of the overall correction. At two levels of wavefront correction based on the SA

(Simulated annealing) algorithm and the SPGD (stochastic parallel gradient descent) algorithm [4], the control elements of wavefront corrector of deformable mirrors are subdivided into four parts depending the location. First of all, with the combination of SA and SPGD algorithm, the entire wavefront is optimized. Then the aberration of corners wavefront will be corrected by HC (Hill Climbing) algorithm [5]. In each step of the corner optimization, the entire surface will be evaluated. The entire wavefront correction effect reaches the ideal level, at the same time the corner of wavefront can be corrected.

The first level of the algorithm is mainly for the optimization of the whole wavefront. The result of SA algorithm is independent of the initial value, and it has the property of asymptotic convergence. The DM unit drive voltage value can converge to the global optimal solution. Then the SPGD algorithm is used to optimize the wavefront aberration to get an exact solution.

The second level of the algorithm is mainly to optimize the corner area of the wavefront. The segment corner area from the whole corrected wavefront and the corresponding drive and the response function is also divided. The local optimum voltage has been obtained by the previous process of the first level and the second level of the algorithm is on this basis. The RMS and PV value is used as the evaluation function and a smaller voltage step is set to increase or decrease the voltage to each of the units in a certain direction. At last, the optimal solution of local area is obtained. The whole wavefront can be evaluated with the change of local voltage and it is one of the important reasons for the use of $\mathrm{HC}$ algorithm in local area correction. Ensure the whole wavefront RMS or PV value is maintained at a reasonable level and the corners can be optimized well.

So it is a two level optimization model, which is from the whole to the local level and the whole wavefront, and it is optimized to the local corner area in the optimization area. The actuator is also the overall move to the local side angle move.

\section{SIMULATION}

\subsection{Simulation Model}

The adaptive optics simulation model of the Sub-regional wavefront correction method is a large aperture 77-element deformable mirror. Fig. 2 shows the structure and actuator-units distribution of this DM and the Fig. 3 shows the response function of the eleventh actuator-unit, which can be measured through interferometer.

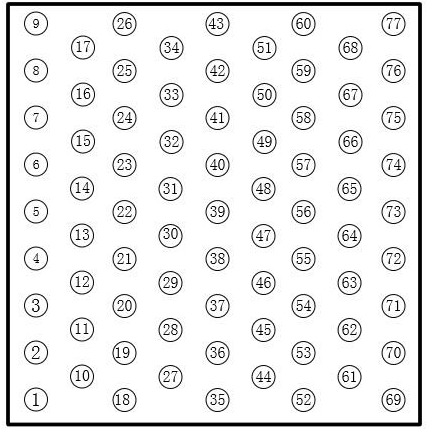

Figure 2. Actuator arrangement of 77-element DM

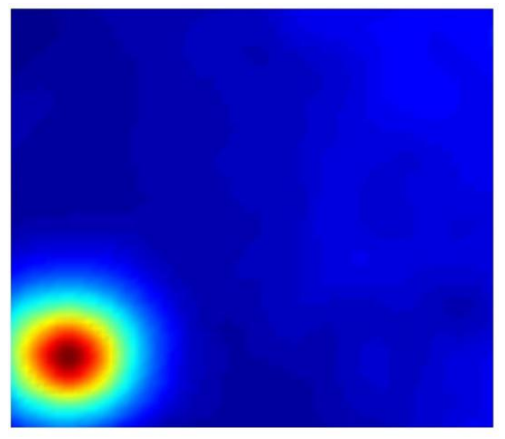

Figure 3. The response function of $10^{\text {th }}$ actuator

Use RMS value as the evaluation function of this system. Zernike mode aberrations of the wavefront are as the input. 


\subsection{Simulation Analysis}

Design the algorithm using the proposed method and the fourth Zernike aberration is used as input to carry out the operation. And the response function of every actuator-unit is measured when the voltage is $20 \mathrm{~V}$. The wavefront is represented by $64 \times 64$ sampling points.

The SA and the SPGD combination algorithm are used in the first level of the whole surface and the defocus is as the input wavefront. SA algorithm is used in the first. The initial temperature of SA algorithm is $\mathrm{T}=200$ and attenuation coefficient is a $=0.95$. Number of calculations is cycle $=10000$, and the RMS value of all surface wavefront is the evaluation function. The global minimum voltage $U$ will be calculated through this algorithm in this situation. The SPGD algorithm is used to optimize the wavefront aberration after optimization of SA algorithm. As a kind of nonmodel optimization algorithm, it is more applicable to the control of more variables. Number of calculations of SPGD algorithm is also cycle $=10000$. So a global optimal solution could be gotten through the combined algorithm, which is a more accurate solution.

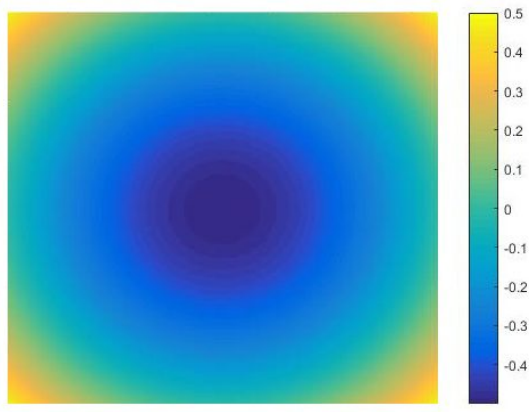

Figure 4. Defocus

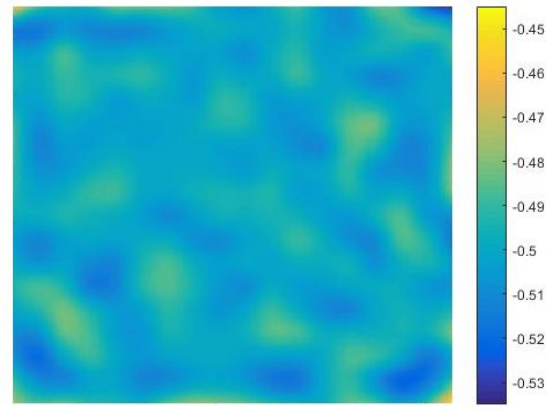

Figure 5. After correction

The initial RMS of focus is 0.2243 . After the completion of wavefront aberration optimization, the final RMS is 0.0072 , $96.8 \%$ lower than the original value. It shows that the first level optimization effect is quite obvious. The second quarter is optimized on the basis of the first level optimization. The optimized wavefront of the lower left corner area is divided up, and eight actuator-units are used as a set.

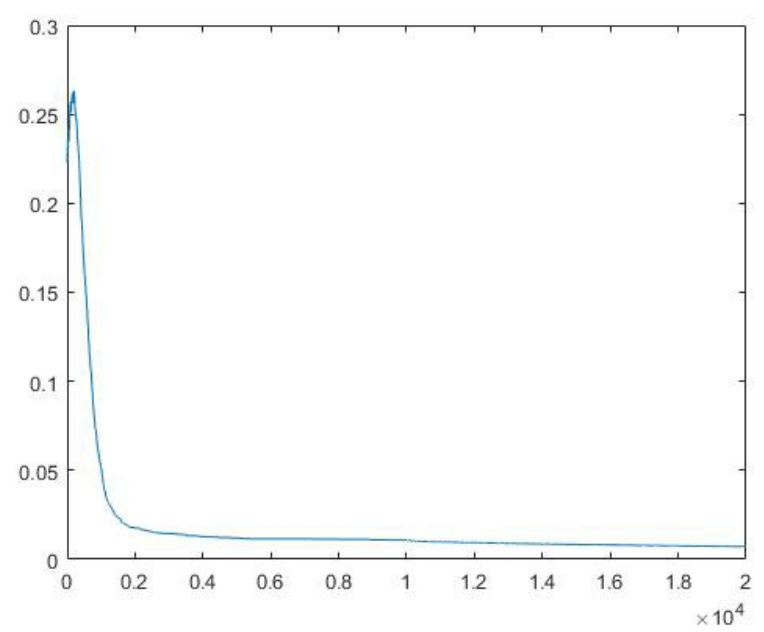

Figure 6. Change of RMS of the first level correction

The original number of the eight units are 1,2,3,10,11,18,19,20. The figure 5 shows the renumbered units. Only the voltage of the eight units are changed in the two stages of optimization process. The response functions of eight units are 
also recalculated and stored. It is an advantage that the length of each voltage change $\Delta u$ is small in HC algorithm. With each voltage change, the evaluation can be carried out. The first step is to determine if this voltage change optimizes the aberration of the edge region. If the aberration of edge area is optimized, then this change will be accepted. The second step is to determine the whole wavefront. If the evaluation of voltage change has too much negative effect on the whole surface, then this change will be discarded.

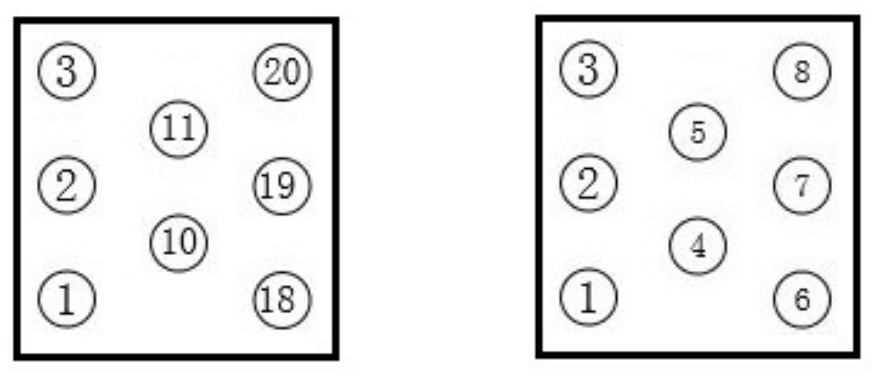

Figure 7. Position and number of actuator in corner area

The second level local $\mathrm{HC}$ algorithm is based on the initial voltage of the eight units of the first level. The step voltage of $\mathrm{HC}$ algorithm is set to $0.2 \mathrm{mv}$. There will be a negative impact on the whole surface if the voltage change is too much. The small step of voltage change is beneficial in maintaining the overall shape. The RMS value of the edge area id reduced from the original 0.0091 to 0.0084 and the reduction range is $7.7 \%$.

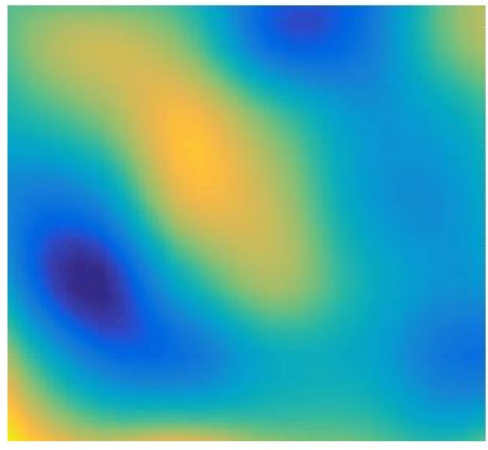

Figure 8. Before the second level correction

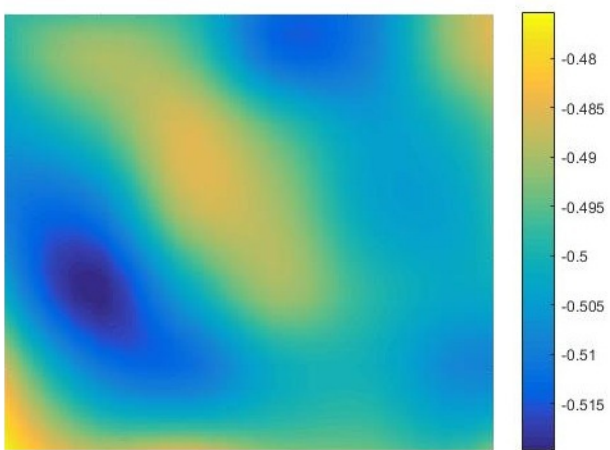

Figure 9. After the correction

The residual error of the whole surface remains unchanged and the aberration of the corner edge is corrected well.

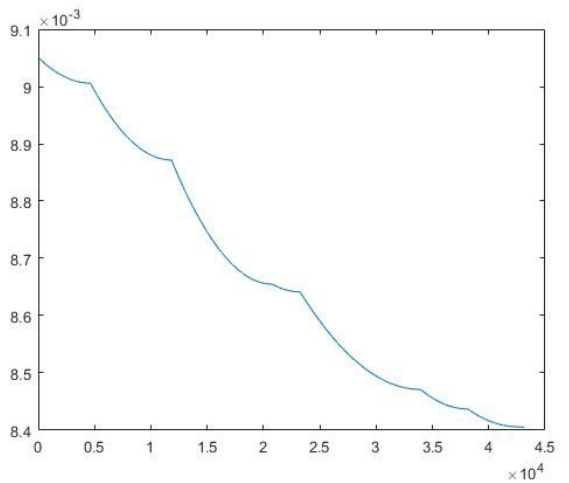

Figure 10. Change of the RMS of corner area

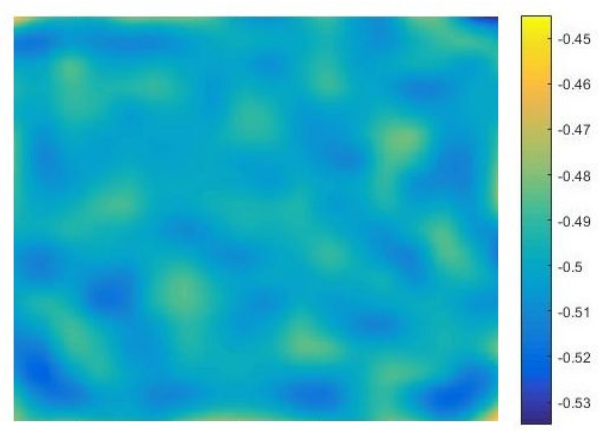

Figure 11. Wavefront after correction 
It can also be analyzed from the results that the correction effect of deformable mirror can be restricted by the structure and driver distribution and the response function of the deformable mirror. But in the other hand, if the structure of deformable mirror is designed properly and the accuracy of the actuators can be guaranteed, the algorithm can get better effect of edge angle optimization.

\section{CONCLUSION}

The novel hybrid algorithm adaptive optics system corrects the distorted wavefront in the sequence from the center parts to the four corners. Aiming at the random aberration, a 77-element AO model based on the method mentioned in this paper is set up, and some kind of aberrations are simulated to research the effect on correct process.

The results reveal that there is a promising prospect for the novel method to be used in corrections of static aberrations. Numerical simulations and simulation correction experiments were conducted, and the results indicated this method can get a high efficiency.

\section{REFERENCE}

[1] Liang, J., D. R. Williams, and D. T. Miller. "Supernormal vision and high-resolution retinal imaging through adaptive optics. " Journal of the Optical Society of America A Optics Image Science \& Vision14.11, 2884-92 (1997).

[2] Tyson, Robert K. "Principles of Adaptive Optics." Physics Today45.45, 261-283 (1991).

[3] Ma, X., et al. "Orientation dependent wavefront correction system under grazing incidence. " Optics Express 21.18, 20497-505 (2013).

[4] Yang, Huizhen, X. Li, and W. Jiang. "High resolution imaging of phase-distorted extended object using SPGD algorithm and deformable mirror."Optical Design and Testing III, 683411-683411-9(2007).

[5] Liu, Y., et al. "Robustness properties of hill-climbing algorithm based on Zernike modes for laser beam correction. " Applied Optics53.10, 140-6 (2014). 